\title{
Streptococcus dysgalactiae subsp. equisimilis: two cases of tonsillitis
}

\author{
Vincenzo Savini', Nicola di Giuseppe², Assunta Manna', Giovanni Di Bonaventura ${ }^{3}$, \\ Chiara Catavitello', Marzia Talia', Andrea Balbinot', Fabio Febbo', Fabio Di Berardino', \\ Domenico D'Antonio' \\ 'Unità Operativa Complessa di Microbiologia e Virologia Clinica, Centro Trasfusionale, Dipartimento di Ematologia, \\ Ospedale "Spirito Santo", Pescara (PE) \\ ${ }^{2}$ Dipartimento di Otorinolaringoiatria e Chirurgia Cervico-Facciale, Ospedale "Spirito Santo", Pescara (PE) \\ ${ }^{3}$ Dipartimento di Microbiologia Clinica, Centro Studi Invecchiamento, Università degli Studi “Gabriele d’Annunzio”, Chieti (CH)
}

Streptococcus dysgalactiae subsp. equisimilis: two cases of tonsillitis

Key Words: Streptococcus, Tonsillitis, Pharyngitis

\section{SUMMARY}

We described two case reports of S. dysgalactiae subsp. equisimilis tonsillitis occurred from January 2005 to January 2007, among patients who come to our observation during these two years.

These patients are paradigmatic of some conditions: adult age, absence of underlying diseases, outbreak of similar pharyngo-tonsillar sympyomatology, unsuccessful oral penicillin therapy, isolation of S. dysgalactiae subsp. equisimilis from throat swab, complete recovery after oral beta-lattamic antibiotic therapy, but total clearance of the microorganism only after oral macrolides administrations. Thus, the intracellular localization of S. dysgalactiae subsp. equismilis, could be in charge of the failure of beta-lattamic antibiotics therapy.

Received May 2, 2007

Accepted May 3I, 2007

\section{INTRODUZIONE}

S. dysgalactiae subsp. equisimilis appartiene al gruppo $\mathrm{C}$ o $\mathrm{G}$ di Lancefield ed è responsabile di rare epidemie di faringotonsillite nelle popolazioni adulta e pediatrica (1); è invece considerato un agente eziologico inusuale di episodi tonsillitici acuti a carattere sporadico (2). Sono documentati tra l'altro episodi di setticemia, endocardite, polmonite, meningite, sostenuti da S. dysgalactiae subsp. equisimilis, che non è pertanto agente eziologico di sole infezioni dell'alto tratto respiratorio $(1,2)$.

Le infezioni faringotonsillari da streptococchi dei gruppi $\mathrm{C}$ e $\mathrm{G}$ di Lancefield sono talora responsabili di incremento del titolo antistreptolisinico e dello sviluppo di sequele non suppurative, in particolare glomerulonefrite ed artrite reattiva $(6,7)$, similmente a quanto ormai noto a proposito delle infezioni da $S$. pyogenes.

Diversamente, gli streptococchi del gruppo B non sono mai stati associati a complicanze non suppurative; tuttavia sono descritte, in corso di infezioni faringotonsillari da $S$. agalactiae, beta-emolitico del gruppo B, localizzazioni silenti o sintomatiche del medesimo stipite a livello cutaneo o genitourinario (2).

Come descritto da altri autori riguardo alle infezioni da $S$. pyogenes, noi riteniamo che una eradicazione faringotonsillare subtotale di $S$. dysgalactiae subsp. equisimilis possa determinare l'insorgenza di recidive e/o di persistente colonizzazione tonsillare e faringea, condizione, quest'ultima, sfavorevole per la comunità, oltre che il paziente stesso.

\section{Tassonomia}

Su terreni al sangue, gli streptococchi beta-emolitici formano tipiche small colonies (Streptococcus anginosus group) o large colonies (Streptococcus pyogenes, gruppo A; Streptococcus equi subsp. zooepidemicus, group C; Streptococcus dysgalactiae subsp. equisimilis, gruppo C o G).

Per quanto $S$. pyogenes e $S$. dysgalactiae siano solitamente considerati streptococchi beta-emolitici, è nota l'esistenza di varianti non-emolitiche (4). Stipiti non-emolitici di S. pyogenes sono stati isolati sia da tonsille di portatori sani quanto da casi di faringotonsillite acuta. In particolare, è stata descritta un'epidemia di faringotonsillite

\section{Corresponding author: Vincenzo Savini}

Unità Operativa Complessa di Microbiologia e Virologia Clinica, Centro Trasfusionale, Dipartimento di Ematologia,

Ospedale "Spirito Santo", via Fonte Romana 8, Pescara (Pe)

Tel.: 3206265740 - 3479440776 - E-mail: vincsavi@yahoo.it 
sostenuta da uno stipite non-emolitico di $S$. pyogenes, associata a sei casi di malattia reumatica.

Per quanto concerne $S$. dysgalactiae, le varianti alfae gamma-emolitiche (non-emolitiche) sono ad oggi raggruppate nella subspecie Streptococcus dysgalactiae subsp. dysgalactiae, mentre gli stipiti betaemolitici, di gruppo $\mathrm{Co} \mathrm{G}$ di Lancefield, fanno parte della subspecie Streptococcus dysgalactiae subsp. equisimilis.

Recentemente sono altresì state descritte varianti non-emolitiche di S. dysgalactiae subsp. equisimilis.

\section{CASI CLINICI}

1. S. R., 30 anni, uomo. Il giorno 08/01/2007 giunge alla nostra osservazione per faringodinia e disfagia intensa, insorte da 3 giorni. Il paziente è apiretico $\left(36.6^{\circ} \mathrm{C}\right)$. L'esame obiettivo evidenzia: discreta presenza di essudato tonsillare bilaterale, ipertrofia tonsillare bilaterale, intenso e diffuso eritema faringotonsillare, ipertrofia dolente di diverse stazioni linfoghiandolari, laterocervicali, bilateralmente. In anamnesi, il paziente riferisce episodio di tonsillite essudativa circa 20 giorni prima, caratterizzato da analoga sintomatologia e trattato con amoxicillina, 1gr x 2/die (ogni 12 ore), per 6 giorni. Il paziente riferisce completa guarigione clinica già al terzo giorno di terapia, con perdurare del benessere per una settimana, quindi recidiva del quadro clinico, che lo ha condotto a rivolgersi alla nostra attenzione.

Il titolo antistreptolisinico è risultato modicamente aumentato (350 UI); negativo è invece il dosaggio di IgM ed IgG anti-Epstein-Barr virus.

Il tampone faringeo ha evidenziato la presenza di numerose colonie di $S$. dysgalactiae subsp. equisimilis. È stata avviata terapia con amoxicillina/acido clavulanico 1 gr x 2/die (ogni 12 ore), per 6 giorni, cui ha fatto seguito pronta e completa remissione della sintomatologia. Un secondo tampone di controllo, eseguito a 1 settimana dall'ultimo giorno di terapia, ha tuttavia evidenziato la persistenza di colonie di $S$. dysgalactiae subsp. equisimilis. Si esegue un secondo ciclo di antibiotico-terapia, identico al precedente. A 7 giorni dal termine dell'assunzione di amoxicillina/acido clavulanico, un terzo tampone faringeo ha documentato ancora la persistenza di colonie di $S$. dysgalactiae subsp. equisimilis. Un secondo dosaggio del titolo anti-streptolisinico, eseguito in concomitanza con il terzo tampone di controllo, ha evidenziato valori complessivamente invariati rispetto alla prima misurazione (340 UI). Il paziente riferisce di aver scrupolosamente aderito alla terapia antibiotica prescrittagli. Si prescrive dunque claritromicina, $500 \mathrm{mg}$ x $2 /$ die (ogni 12 ore), per 6 giorni. A 7, 14 e 21 giorni dal termine della terapia con macrolide, i tamponi faringei di controllo non evidenziano più presenza di colonie di S. dysgalactiae subsp. equisimilis ed il dosaggio del titolo antistreptolisinico risulta rientrato entro il range di normalità.

Nessuno dei tamponi faringei eseguiti ha evidenziato colonie di lieviti, né colonie streptococciche gamma-emolitiche.

Le colonie di streptococchi alfa-emolitici isolati si sono dimostrate appartenere alle specie Streptococcus mitis e Streptococcus oralis.

2. A. C., 32 anni, donna. Il giorno 03/11/2006 giunge presso il nostro dipartimento con richiesta di esecuzione di tampone faringeo. La paziente, apiretica $\left(36.5^{\circ} \mathrm{C}\right)$, riferisce insorgenza, circa 15 giorni prima, di intense faringodinia e disfagia, lieve ipoacusia, notevole ipertrofia tonsillare e "sensazione di doloroso igombro alla gola". In assenza di essudato tonsillare, il quadro clinico descritto era stato attribuito a faringotonsillite virale e trattata con soli antisettici topici in spray e pastiglie. Al persistere della sintomatologia, e a fronte del rilievo di un movimento del titolo antistreptolisinico (360 UI), la paziente giunge alla nostra osservazione per esecuzione di un tampone faringeo. Quest'ultimo evidenzia la presenza di numerose colonie di Streptococcus dysgalactiae subsp. equisimilis. L'ispezione faringea evidenzia intensa e diffusa iperemia faringotonsillare, considerevole ipertrofia tonsillare, bilaterale, in assenza di essudato visibile, ed ipertrofia di alcune stazioni linfoghiandolari laterocervicali. Il condotto uditivo e la membrana timpanica appaiono nella norma, bilateralmente.

È stata avviata terapia con amoxicillina/acido clavulanico, 1 gr x 2/die (ogni 12 ore), per 6 giorni, cui ha fatto seguito remissione completa del quadro clinico. Un tampone faringeo di controllo, a 1 settimana dal termine della terapia, ha evidenziato la persistenza di colonie di $S$. dysgalactiae subsp. equisimilis. Si esegue un secondo ciclo di 6 giorni di amoxicillina/acido clavulanico, al medesimo dosaggio del precedente. A 7 giorni dal termine del trattamento, un terzo tampone faringeo documenta la persistenza di colonie di $S$. dysgalactiae subsp. equisimilis; parallelamente, un nuovo dosaggio del titolo antistreptolisinico evidenzia valori sostanzialmente invariati dello stesso (360 UI). La paziente riferisce la scrupolosa adesione alla terapia prescrittale. Si avvia terapia con claritromicina, $500 \mathrm{mg} \times 1 /$ die, formulazione a rilascio modificato (ogni 24 ore), per 6 giorni. A 7, 14 e 21 giorni dal termine della somministrazione di macrolide, i tamponi di controllo evidenziano la completa scomparsa di $S$. dysgalactiae subsp. equisimilis, associata a rientro del titolo antistreptolisinico nell'intervallo di normalità. Anche in questo caso, nessuno dei tamponi 
faringei ha evidenziato la presenza di colonie streptococciche non-emolitiche. Gli streptococchi viridanti isolati appartenevano alle specie $S$. mitis e $S$. oralis. Negativa è risultata la ricerca di $\operatorname{IgM}$ ed IgG anti-EBV.

\section{MATERIALI E METODI}

I tamponi faringei sono stati seminati su Bloodsheep agar, CNA agar, Mannitol-salt agar (onde escludere la presenza di Staphylococcus aureus, la cui sovrainfezione di reisidui alimentari annidati nelle cripte tonsillari potrebbe mimare l'induito purulento da infezione streptococcica o l'essudazione tipica della mononucleosi infettiva) e Sabouraud-dextrose agar (al fine di escludere una candidosi faringotonsillare).

Il Blood-sheep agar ed il CNA agar sono stati incubati per $48 \mathrm{~h}$, a $37^{\circ} \mathrm{C}$, in aerobiosi ed in atmosfera al $5 \%$ di $\mathrm{CO}_{2}$ ed esaminati a 24 e 48 ore. Dopo 24 ore era già apprezzabile la crescita di colonie streptococciche beta-emolitiche, in ambedue le condizioni di incubazione, su ambedue $\mathrm{i}$ terreni al sangue.

Sono state eseguite cinque subcolture seriali degli isolati, sia su Blood-sheep agar che su CNA agar, al fine di stabilire se l'attività emolitica osservata fosse realmente una caratteristica stabile, come in effetti si è dimostrato.

Non sono state osservate colonie di $S$. aureus (dopo 3 giorni di incubazione), né di Candida spp. (dopo 4 giorni di incubazione).

Rare colonie alfa-emolitiche si sono sviluppate su ambedue i terreni al sangue ed in entrambe le condizioni di incubazione.

Le colonie di streptococco beta-emolitico sono state identificate biochimicamente come Streptococcus dysgalactiae subsp. equisimilis (Vitek 2 automatic system for biochemical identification of bacteria and yeasts, bioMérieux).

L'esecuzione del test di agglutinazione al lattice con antisieri dei gruppi A, B, C, D, F e G (Streptococcal grouping kit, Oxoid) ha consentito di attribuire l'isolato al gruppo $\mathrm{C}$ di Lancefield.

Le colonie alfa-emolitiche isolate sono state identificate biochimicamente come Streptococcus oralis e Streptococcus mitis (Vitek 2 automatic system for biochemical identification of bacteria and yeasts, bioMérieux) e ciò al fine di escludere che la presenza di Streptococcus salivarius potesse inibire la crescita di streptococchi beta-emolitici, all'interno del raggio di azione delle batteriocine rilasciate da $S$. salivarius nel terreno di coltura (4).

\section{CONCLUSIONI E DISCUSSIONE}

Sebbene sottovalutate, le faringotonsilliti costituiscono un considerevole problema clinico, a causa della elevata incidenza, della notevole frequenza di recidive, a breve e lungo termine, delle complicanze suppurative (ascessi tonsillari) e non suppurative (malattia reumatica, glomerulonefrite, artralgie ed artriti, cardite) complicanti talora l'infezione tonsillare streptococcica e, dunque, della cospicua spesa sanitaria che ne consegue.

Il $30 \%$ dei casi pediatrici riconosce un'eziologia batterica, laddove il $90 \%$ dei casi osservati nella popolazione adulta sono verosimilmente di origine virale o riconducibili ad altri fattori, quali infezioni fungine o patologie settali e/o rinosinusali (in cui è usuale il riscontro di respirazione notturna a bocca aperta, con conseguente secchezza faringea mattutina e diurna, eritema faringeo e faringodinia), reflusso gastroesofageo (con conseguente flogosi faringea da succhi gastrici).

Streptococcus pyogenes è certamente il principale agente eziologico di infezione tonsillare batterica. Gli streptococchi beta-emolitici dei gruppi B, C e $\mathrm{G}$ di Lancefield sono sovente presenti quali commensali della mucosa faringea umana, sebbene giochino talvolta un ruolo patogeno nell'insorgenza di faringotonsilliti essudative $(1,2)$, simili a quelle causate da streptococchi di gruppo A.

Inoltre, casi relativamente rari di faringotonsillite sono dovuti a germi di riscontro meno frequente, quali Streptococcus pneumoniae, Neisseria spp., Corynebacterium spp., Chlamydia pneumoniae, Mycoplasma pneumoniae, spesso in associazione a flora batterica anaerobia (5).

Streptococcus dysgalatiae subsp. equisimilis rappresenta un agente eziologico infrequente di faringotonsillite sporadica, nell'adulto e nel bambino. Nonostante tale microrganismo formi generalmente tipiche large colonies beta-emolitiche, sono noti stipiti deficitari dell'enzima emolisina, formanti colonie gamma-emolitiche (non-emolitiche), come descritto anche per $S$. pyogenes (4). Dunque, è in primo luogo fondamentale evitare l'errore di considerare a priori le eventuali colonie non-emolitiche quali isolati appartenenti alla flora commensale orofaringea. Al contrario, riteniamo opportuno ottenere colture pure delle stesse, onde identificarne biochimicamente il genere, la specie ed eventualmente la subspecie. Il legame tra infezione da $S$. dysgalactiae subsp. equisimilis emolisina-privo ed eventuale insorgenza di artralgie, artriti o ematuria, rimarrebbe misconosciuto qualora il laboratorio refertasse l'assenza di colonie beta-emolitiche come campione negativo.

È dunque inopportuno refertare l'esclusiva presenza di $S$. pyogenes, o di streptocchi beta-emolitici come streptococchi di gruppo A o non-A. Infatti, streptococchi beta-emolitici dei gruppi $\mathrm{C}$ e $\mathrm{G}$ di Lancefield possono essere responsabili di sequele non suppurative post-tonsillite, similmente a quanto noto per $S$. pyogenes, oltre che dell'in- 
cremento del titolo anti-streptolisinico.

Diversamente, in corso di infezione da $S$. dysgalactiae, di gruppo $\mathrm{B}$, non sono attese sequele non suppurative ad interessamento articolare o renale, ma l'attenzione verrà posta sulla eventuale copresenza di infezioni a distanza sostenute dal microrganismo medesimo. Tali pazienti andrebbero inoltre studiati per quanto concerne le abitudini sessuali, onde stabilire un eventuale legame tra localizzazione faringea e genitourinaria. Al contrario, la comparsa di interessamento articolare o un concomitante incremento del TAS dovrebbe indurre a considerare l'ebentualità di una sovrapposta infezione da streptococchi dei gruppi A, C o $\mathrm{G}$ (eventualmente emolisina-privi), o di malattie concomitanti (infezioni virali, artralgie da febbre, patologie ortopediche o reumatologiche, altro), laddove $S$. agalactiae potrebbe rappresentare, nel caso ipotizzato, un semplice colonizzante. Ciò a sottolineare l'importanza di una corretta identificazione delle colonie beta- e gamma-emolitiche isolate da un tampone faringotonsillare.

La presenza di induito biancastro sulle tonsille, resitente al trattamento antibiotico, andrebbe posto in diagnosi differenziale con un'infezione da Epstein-Barr virus o con candidiasi delle mucose orofaringee e non attribuito necessariamente ad un'infezione streptococcica particolarmente resistente agli antimicrobici. La sovrainfezione da Candida spp. può infatti complicare una faringotonsillite virale, ponendoci di fronte ad un quadro febbrile (per infezione virale) con presenza di essudato bianco-grigiastro sulle tonsille (da sovrapposizione fungina), a mimare un quadro di infezione streptococcica.

Il solo riscontro di eritema faringeo è invece solitamente da attribuirsi ad infezione virale (alcuni Adenovirus persistono per anni nel tessuto linfoide tonsillare), ma riteniamo che la presenza di una flogosi particolarmente spiccata, associata a notevole faringodinia e disfagia, in special modo se associata ad interessamento linfoghiandolare, anche in assenza di febbre e di essudato tonsillare, debba far sorgere il sospetto di un'eziologia batterica; l'infezione da streptococchi di gruppo A, C e G, infatti, non sempre dà luogo ad evidente essudazione. Un tampone faringeo, correttamente eseguito, e la pronta terapia antibiotica, andrebbero dunque prese in considerazione, tenendo presente che il rischio di sequele non suppurative grava anche sulle infezioni da streptococchi dei gruppi $\mathrm{C}$ e $\mathrm{G}$, in particolare $S$. dysgalactiae subsp. equisimilis, seppur in misura inferiore rispetto a Streptococcus pyogenes.

Penicillina e derivati sono ad oggi il golden standard nel trattamento delle faringotonsilliti da streptococchi beta-emolitici (9), in virtù della usuale completa sensibilità di questi ultimi alle beta-lattamine orali e parenterali solitamente impiegate. Tuttavia, numerosi episodi di tonsilliti da $S$. pyogenes o $S$. dysgalactiae subsp. equisimilis recidivano o non vanno incontro a remissione completa, nonostante terapia con penicillina o cefalosporina orale o parenterale e la completa adesione del paziente al trattamento. In buona parte dei casi, ciò è da attribuirsi alla presenza in faringe delle beta-lattamasi prodotte dalla flora microbica commensale.

Riteniamo pertanto opportuno ricorrere all'impiego di amoxicillina/acido clavulanico o ampicillina/sulbactam, piuttosto che di sola amoxicillina o ampicillina, a fronte della dimostrata sensibilità in vitro di quest'ultime. Inoltre, studi recenti hanno dimostrato che $S$. pyogenes, solitamente considerato un patogeno extracellulare, può internalizzare nell'epiteliocita faringeo, divenendo inattaccabile dalle molecole beta-lattamiche (8).

Similmente, alla luce dei due casi clinici descritti, noi ipotizziamo che $S$. dysgalactiae subsp. equisimilis possa parimenti agire come patogeno extracellulare ed intracellulare e ciò giustificherebbe l'incompleta risposta alla somministrazione di penicilline o cefalosporine orali o parenterali, o la completa risposta clinica ma in assenza di eradicazione totale del microrganismo. Ciò può condurre a recidiva di faringo-tonsillite, a breve, medio o lungo termine, e/o all'instaurarsi della condizione di portatore, come descritto in letteratura a proposito di S. pyogenes. Sono noti tra l'altro casi di faringo-tonsillite essudativa da streptococchi di gruppo $\mathrm{G}$ dovuti a cibo contaminato (3), che potrebbe pertanto costituire un veicolo inusuale di trasmissione dell'infezione, accanto alla più frequente modalità per via aerea: la contaminazione alimentare e la presenza di portatori costituirebbero seri problemi, in particolare in asili, mense, scuole, collegi, comunità chiuse. $S$. dysgalactiae subsp. equisimilis potrebbe internalizzare nelle cellule tonsillari o della mucosa faringea o persistere in seno ai fagociti mediante meccanismi di resistenza alla fagocitosi, il che spiegherebbe la clearance subtotale del microrganismo avutasi anche dopo ripetuti cicli di beta-lattamina, nei due casi clinici osservati.

Di fronte al dato clinico e microbiologico di faringotonsillite da $S$. dysgalactiae subsp. equismilis, pensiamo pertanto sia opportuno non accontentarsi della sola risoluzione dei sintomi, ma far seguire alla terapia standard con beta-lattamici un tampone faringeo di controllo, anche in presenza di completa remissione sintomatologica, onde assicurarsi della completa eradicazione del patogeno; si ricorrerà all'impiego di macrolide (claritromicina, azitromicina), ad attività extra- ed intracellu- 
lare, in caso di clearance incompleta del microrganismo $(9,10)$. Qualora vi sia persistente colonizzazione faringea, nonostante terapia con betalattamici, ed impossibilità di utilizzo del macrolide per dimostrata resistenza del ceppo in esame (10), o diatesi allergica del paziente a tali chemioterapici, andranno prese in considerazione molecole alternative (es. levofloxacina, moxifloxacina, doxiciclina, clindamicina), dotate di attività intracellulare.

\section{BIBLIOGRAFIA}

1. Benjamin J, Perriello VA. Pharyngitis due to group C hemolytic streptococci in children. J Pediatr, 1976; 89: 254-5.

2. Chowdhury MNH, Kambal AM, Al-Eissa YA, Khaliq MRA, Al-Ayed IH, Al-Sanie AM. Non-group A streptococci: are they pathogens in throat? J R Soc Health, 1997; 117: 160-3.

3. Cohen D, Ferne M, Rouach T, Bergner-Rabinovitz S. Foodborne outbreak og group $G$ streptococcal sore throat in an Israeli military base. Epidemiol Infect,
1987; 99: 249-5.

4. Dierksen KP, Tagg JR. Haemolysin-deficient variants of Streptococcus pyogenes and S. dysgalactiae subsp. equismilis may be overlooked as aetiological agents of pharyngitis. J Med Microbiol, 2000; 49: 811-6.

5. Esposito S, Blasi F, Bosis S, et al. Aetiology of acute pharyngitis: the role of atypical bacteria. J Med Microbiol, 2004; 53(Pt 7): 645-51.

6. Gnann JW, Gray BM, Griffin FM, Dismukes WE. Acute glomerulonephritis following group G streptococcal infection. J Infect Dis, 1987; 156: 411-2.

7. Jansen TLTA, Janssen M, de Jong AJL. Reactive arthritis associated with group $\mathrm{C}$ and group $\mathrm{G}$ betahemolytic streptococci. J Rheumatol, 1998; 25: 112630 .

8. Neeman R, Keller N, Barzilai A, Koreman Z, Sela S. Prevalence of internalisation-associated gene, prtF1, among persistent group-A streptococcus strains isolated from asymptomatic carriers. Lancet, 1998; 352: 1974-7.

9. Pichichero ME, Casey JR, Mayes T, et al. Penicillin failure in streptococcal tonsillopharyngitis: causes and remedies. Pediatr Infect Dis J, 2000; 19(9): 91723.

10. Tarlow MJ. Macrolides in the management of streptococcal pharyngitis/tonsillitis. Pediatr Infect Dis J, 1997; 16(4): 444-8. 